Published in final edited form as:

Hand Clin. 2015 May ; 31(2): 205-215. doi:10.1016/j.hcl.2014.12.002.

\title{
Management of Complications of Distal Radius Fractures
}

\author{
Kevin C. Chung, MD, MS ${ }^{1}$ and Alexandra L. Mathews, BS $^{2}$ \\ ${ }^{1}$ Professor of Surgery, Section of Plastic Surgery, Assistant Dean for Faculty Affairs, The \\ University of Michigan \\ ${ }^{2}$ Research Assistant, Section of Plastic Surgery, Department of Surgery, The University of \\ Michigan Health System
}

\section{Synopsis}

Treating a fracture of the distal radius may require the surgeon to make a difficult decision between surgical treatment and nonsurgical management. The use of surgical fixation has recently increased owing to complications associated with conservative treatment. However, conservative action may be necessary depending on certain patient factors. The treating surgeon must be aware of the possible complications associated with distal radius fracture treatments to prevent their occurrence. Prevention can be achieved with a proper understanding of the mechanism of these complications. This article discusses the most recent evidence on how to manage and prevent complications following a fracture of the distal radius.

\section{Keywords}

preventive; complications; early diagnosis; CRPS; malunion; infection; extensor tendon; flexor tendon

\section{Introduction}

Fractures occurring at the distal end of the radius are seen frequently in emergency departments, representing approximately one sixth of all fractures [1]. Based on decades of extensive research, surgeons have developed multiple approaches for the treatment of distal radius fractures, including both conservative and non-conservative options. These options include closed reduction and casting, closed reduction and percutaneous pinning, external fixation, and open reduction with internal fixation (ORIF) [2]. The conservative treatment of closed reduction and casting has historically been the mainstay of treatment for distal radius fractures, however, owing to the increased complication rate associated with this treatment

(C) 2015 Published by Elsevier Inc.

Corresponding author and reprint requests sent to: Kevin C. Chung, MD, MS, Section of Plastic Surgery, The University of Michigan Health System, 1500 E. Medical Center Drive, 2130 Taubman Center, SPC 5340, Ann Arbor, MI 48109-5340, Phone: 734-936-5885, Fax: 734-763-5354, kecchung@med.umich.edu.

Publisher's Disclaimer: This is a PDF file of an unedited manuscript that has been accepted for publication. As a service to our customers we are providing this early version of the manuscript. The manuscript will undergo copyediting, typesetting, and review of the resulting proof before it is published in its final citable form. Please note that during the production process errors may be discovered which could affect the content, and all legal disclaimers that apply to the journal pertain. 
such as fracture collapse, surgical options, specifically ORIF, are becoming more common $[1,3]$.

Overall, distal radius fracture complications have been found to occur in as little as $6 \%$ of patients and as many as $80 \%$ of patients, pending on the definition of complication [4]. Complications after distal radius fractures occur for many reasons, and often vary depending on the method of treatment [2]. When deciding on a treatment option, it is important that surgeons focus on recognition, management, and prevention of known associated complications in order to achieve a good outcome [5]. Patient factors must also be taken into account when considering treatment methods. Factors including patient lifestyle, age, mental attitude, social support, comorbid conditions, and compliance with treatment can influence the likelihood for complications [1]. For example, a prospective cohort study designed to identify predictors of hand outcomes after distal radius fracture treatment found that increased age and lower income led to a significantly worse long-term outcome 1 year after successful surgery using a volar locking plating system [6]. Awareness of potential risk factors can aid in the prevention of possible complications. This review will focus on the prevention and management of complex regional pain syndrome, malunions, infections, and tendon complications after distal radius fracture treatment.

\section{Complex Regional Pain Syndrome}

Characterized by autonomic dysfunction, trophic changes, and impaired function, complex regional pain syndrome (CRPS) can occur after both operative and non-operative treatments of a distal radius fracture [7]. The rate of incidence of CRPS after a fracture of the distal radius has been found to vary (1\%-37\%) [8-10], and often rises with increasing severity of the fracture [11]. There is no definitive cause or treatment for this syndrome; however, many association factors have been discovered. For operatively treated fractures, excessive distraction with an external fixator can raise the risk of CRPS development [12]. For fractures treated non-operatively, a correlation was found between an increased incidence of CRPS and an increase in pressure under the cast [13]. There are also several theories for the pathophysiological mechanism leading to the development of CRPS; however, the true cause remains unclear. Possible involvement of the sympathetic nervous system, abnormal inflammatory reactions, sequelae of nerve injury, and psychological disturbances have all been considered [14].

CRPS can be categorized into two different types depending on the presence or absence of nerve trauma. Formerly known as reflex sympathetic dystrophy, CRPS type I is defined as chronic pain without an identifiable nerve injury [7]. Conversely, CRPS type II is characterized by nerve involvement. It has been reported that women, the elderly, and individuals with a psychological predisposition have a higher likelihood of developing CRPS type I [15]. For example, Roh et al recently evaluated potential factors influencing the rate of CRPS type I after the surgical treatment of a distal radius fracture, and found female patients to be 2.2 times more likely to develop CRPS type I compared to male patients [11]. Additionally, incidences of CRPS have been shown to be higher in smokers compared to nonsmokers [16]. 
Early diagnosis is important for ensuring the best possible recovery. Patients with suspected CRPS can begin to show symptoms as early as 2 weeks after surgery, but symptoms can also develop several weeks after surgery [14]. Early diagnosis and treatment for CRPS has been shown to result in recovery in 80-90\% of cases [14]. However, diagnosing CRPS can prove to be difficult because there are no formal, standardized diagnostic criteria available to date. Zyluk et al. highlighted the recent increase in use of the International Association for the Study of Pain (IASP) criteria of diagnosis in many scientific studies [14]. The IASP criteria involves 4 categories for diagnosing CRPS including sensory, vasomotor, sudomotor/edema and motor trophic [17].

In a clinical setting, patients with possible symptoms of CRPS usually present with pain, swelling, and changes in color, temperature, and perspiration of the affected limb [14]. Pain is normally described as a tearing or burning sensation and is often intensified by exposure to cold [18]. We often observe a shiny appearance of the upper extremity as an indication of CRPS (Figure 1). The absence of pain relief after narcotic use is also a good indication for possible CRPS, as patients with this condition are typically unresponsive to this form of pain medication [18]. Other indications that require careful consideration of possible CRPS include patients with unexpected intense pain, stiffness, sleep difficulties or slower than anticipated recovery [18].

Certain diagnostic tests have been found to aid in the diagnosis of CRPS. Radiographic images can display irregularities in patients with CRPS; however, changes may not be visible until 2 or more weeks post injury [18]. Radiographs may show osteopenic bone with subchondral and periarticular resorption, but normal results may be found in as many as $30 \%$ of patients with CRPS symptoms [18]. Additionally, phase I and phase II bone scans may show hyperperfusion (hot or warm hand) and hypoperfusion (cold, stiff hand), which are common symptoms of patients with CRPS [7]. Three-phase bone scans demonstrate a high specificity and are useful when confirming a positive diagnosis of CRPS; however, a poor correlation between three-phase bone scans and CRPS has been described due to a reported sensitivity of only 50\% [19].

Given the high prevalence of CRPS after fractures of the wrist, researchers have studied possible prevention techniques including the use of vitamin C. Two studies by Zollinger and colleagues assessed the relationship between vitamin $\mathrm{C}$ use and the incidence of CRPS for patients with non-operatively treated wrist fractures and concluded that vitamin $\mathrm{C}$ reduced the prevalence of CRPS [20,21]. A more recent double-blind prospective randomized study of patients treated both operatively and non-operatively for distal radius fractures provided further support for the use of vitamin C [22]. Shah et al. found that 500mg of vitamin C taken daily for fifty days after a distal radius fracture lowered the risk of CRPS from $10 \%$ to $2 \%$ [22]. The use of vitamin C was found to have no effect on patients given a dose of $200 \mathrm{mg}$ or less per day or greater than $500 \mathrm{mg}$ per day. Researchers suggested that $500 \mathrm{mg}$ should be the recommended amount and that typical dietary intake without the addition of a vitamin C supplement may be insufficient in the prevention of CRPS [22]. Excessive amounts of vitamin $\mathrm{C}$ can result in side-effects including diarrhea, abdominal cramping, and kidney stone formation [22]. Vitamin C should be given with caution, especially to patients 
who have been diagnosed with hyperoxaluria or hyperuricosuria due to the risk of renal calculi development [5, 22].

These studies [20-22] influenced the decision by the American Academy of Orthopedic Surgeons (AAOS) in 2009 to recommend the use of vitamin C in the prevention and management of CRPS following distal radial fractures [23]. However, a recent randomized controlled trial by Ekrol et al contradicted this recommendation after finding no significant difference in the incidence of CRPS with the use of vitamin $\mathrm{C}$ after a distal radius fracture [24]. Based on these results, the use of vitamin $C$ may not have strong scientific evidence to endorse its use.

After a positive diagnosis of CRPS following a distal radius fracture, a multidisciplinary treatment approach is recommended. A combination of psychiatric therapy, occupational therapy, and pain management therapy has been shown to produce the best recovery outcome [18]. Passive and active range of motion, splinting, and contrast baths may be performed along with oral medication as a first line of treatment [18]. Based on clinical experience, specific classes of oral agents are recommended such as antidepressants, adrenergic agents, anticonvulsants and membrane stabilizing agents [7]. Li et al. recently explained how treatment for CRPS can often depend on the patient's presentation of symptoms. For a hot, swollen hand, the investigators recommend an antidepressant in combination with an anticonvulsant [7]. Conversely, for patients presenting with cold, stiff hands, a mild antidepressant in combination with a calcium channel blocker is suggested [7]. Other possible treatments for early forms of CRPS include calcitonin, physiotherapy, or free radical scavengers. Researchers developed a free radical scavengers treatment using the assumption that CRPS is caused by an exaggerated inflammatory response to trauma, medicated by an overproduction of toxic oxygen and hydroxyl free radicals [25]. When diagnosed early, patients may expect a significant reduction in pain within 1 month of treatment [14].

Intravenous agents have also been used to treat CRPS following a distal radius fracture; however, there is controversy in recent literature on the effectiveness of agents such as guanethidine, cortisone, reserpine, lidocaine, and bretylium [7]. Livingstone et al. studied this relationship by randomizing 57 patients diagnosed with CRPS type I nine weeks after an isolated closed distal radius fracture to receive either serial intravenous regional blockade with guanethidine or a normal saline solution [26]. They found no significant analgesic advantage for the treatment of early CRPS type I after a distal radius fracture and concluded that this treatment may even delay the resolution of vasomotor instability [26]. Paraskevas and colleagues found results conflicting with Livingston et al. after evaluating the efficacy of both guanethidine and lidocaine in the treatment of CRPS of the hand [26, 27]. Seventeen of the 28 patients evaluated reported excellent pain relief and full restoration of both function and range of motion of the affected hand [27]. Further studies should be performed on the use of intravenous regional blockade in patients diagnosed with CRPS following a distal radius fracture in order to clarify this discrepancy. 
A malunion is the most common complication that occurs following a fracture of the distal radius [28]. A malunited distal radius fracture can be extra-articular, intra-articular or both [29] and occurs when a fracture heals with improper alignment, articular incongruity, incorrect length, or a combination of these elements [30]. Extra-articular malunions are commonly characterized by a loss of the normal palmar tilt of the articular surface in the sagittal plane, a loss of ulnar inclination in the frontal plane, and a loss of length relative to the ulna [29]. Conversely, an intra-articular malunion is frequently associated with a step-off or a gap at the radiocarpal joint and/or the distal radioulnar joint [29].

Researchers have found many indications for the development of distal radius fracture malunions. Fractures treated conservatively are a common cause of malunions, For example, a recent study comparing the outcomes and complications of distal radius fractures between volar plating and non-operative treatment, found that $35 \%$ of the patients treated conservatively developed a malunion [31]. Patients treated with volar plate fixation, however, reported no incidence of malunion [31]. In 2010, the AAOS aimed to prevent possible distal radius fracture complications associated with conservative treatment by publishing guidelines for operative fixation. According to these guidelines, distal radius fractures displaying a post-reduction dorsal tilt of $>10^{\circ}$, radial shortening of $>3 \mathrm{~mm}$, or intraarticular displacement or step-off of $>2 \mathrm{~mm}$ indicate a need for surgical fixation [23]. Malunions can also be anticipated in patients with a dorsally displaced fracture when the strongest portion of the metaphysis, the volar cortex, is not in contact [30].

Malunited distal radius fractures are associated with a variety of symptoms including decreased grip strength, decreased wrist mobility, increased pain, and worsened cosmetic appearance [29, 32]. Patients may also present with pain over the ulnar wrist, caused by either a distal radio-ulnar joint (DRUJ) incongruity or an ulna impaction [30]. Patients treated in our facility commonly complain of decreased motion at the wrist, specifically pronation and supination, in addition to chronic pain. Pronation and supination deformities can be seen in both dorsally and palmarly angulated malunions [33].

A suspected malunion should be confirmed with radiographic images and verified with specific criteria used to define a malunion of the distal radius. The criteria used in our facility involve: [30]

1. Radial inclination $<10$ degrees

2. Volar tilt $>20$ degrees, dorsal tilt $>20$ degrees

3. Radial height $<10 \mathrm{~mm}$

4. Ulnar variance $>2+$

5. Intra-articular step or gap $>2 \mathrm{~mm}$

Evidence recommends surgeons take into account the patient's motivation, functional demands, and the anatomy of the deformity before deciding on a treatment option for a distal radius malunion [29]. Surgical correction should be strongly considered for patients 
presenting with both clinical and radiographic symptoms of a malunion [30]. Fernandez recommended a corrective osteotomy if the angulation of the distal articular surface of the radius became greater than 25 degrees in the sagittal plane [34].

If surgical correction is agreed upon by the patient and surgeon, pre-operative planning should be executed. Pre-operative anteroposterior, lateral, and oblique x-rays of the wrists are used to determine the specific fracture pattern (Figure 2). The malunited distal radius fracture should be compared to the contralateral normal wrist, allowing the surgeon to identify the proper length, angle extent, and type of osteotomy needed [28]. Additionally, CT scans and 3D imaging can be helpful in determining malunion alignment and aid in the reconstructive planning, and are used in our facility. Researchers have noted the successful use of computer-assisted techniques in the evaluation, deformity modeling, preoperative planning, and intraoperative execution of osteotomies [35-37].

A corrective osteotomy should be performed as soon as possible after the diagnosis to correct the biomechanics of the wrist and prevent or minimalize possible soft tissue contracture [30]. By using an osteotomy to adjust the alignment of the distal end of the radius in relation to the wrist and the distal end of the ulna, preliminary reduction can be achieved. Corrective osteotomies have also been shown to improve wrist and forearm motion as well as patient pain levels [38]. Jupiter and Ring retrospectively compared patients treated with either early or late osteotomies, documenting considerable positive results after early reconstruction as well as a decrease in the over-all period of disability [39].

The key factor of the surgical technique for corrective osteotomies is placing the wedge osteotomy through the fracture to recreate the initial fracture pattern [30]. Two types of osteotomies are used, each offering specific advantages and disadvantages. A closing wedge osteotomy allows direct bone-to-bone contact and offers more stability, preventing the need for bone grafting as well as the potential for nonunions [28]. However, this technique can cause the distal radius to become shortened relative to the ulna. Closing wedge osteotomies are often paired with an additional osteotomy, shortening the ulna to maintain the DRUJ. Wada et al. presented encouraging results using a radial closing wedge osteotomy when correcting a dorsal tilt deformity of the distal radius, simultaneously pairing the procedure with an ulnar-shortening osteotomy [40]. However, if the shortened distal radius is not addressed, ulnar abutment can occur, resulting in impaction of the ulnar head against the carpus and gradual development of TFCC lesions and ulnocarpal arthrosis [41].

Opening wedge osteotomies are more popular because they restore the radial length preventing the need for a distal ulna procedure [28]. This technique can also correct angular deformities in both the frontal and sagittal planes. The disadvantage of opening wedge osteotomies is the risk of increased instability of the construct before it has healed completely [28]. There is also a possibility of construct failure from axial loading associated with early wrist motion [28].

After the biomechanics of the wrist have been corrected with an osteotomy, the distal radius fracture must be fixated. The surgical fixation approach depends greatly on the fracture 
pattern and the preference of the surgeon. However, the use of a volar approach has increased recently as an alternative to dorsal plate fixation and external fixation $[42,43]$. The volar approach was introduced to promote prevention of complications related to the dorsal approach, especially extensor tendon injuries [44]. Approaching the fracture from a volar aspect permits more space for plate fixation and the concave surface protects the flexor tendons from hardware irritation [45-47]. The flexor tendons are located far from the volar surface and placement of the pronator quadratus over the volar plate has been suggested to prevent irritation [44]. Volar plate fixation has also been found to result in a shorter period of immobilization and an earlier return to previous activity level [31].

Additional advantages of volar locking plates include the decreased requirement for bone grafting. Traditionally, the use of an iliac crest structural bone graft and fixation with a dorsal plate has been recommended for substantial bone gaps greater than $1 \mathrm{~cm}$ [48]. However, donor-site morbidity has been reported in as high as $20 \%$ of cases, along with other minor complications [49]. Researchers began to study the use of dorsal plate fixation without the addition of bone grafting. This was found to be an effective technique [48], however, the high incidence of plate removal due to painful hardware, tendon irritation or tendon rupture led to the increased use of volar plates [50]. Haase and Chung have shown that the volar approach restores a sturdy volar cortex where a distal plate can be placed, avoiding the need for a large bone graft [30]. Tarallo et al. and Mahmoud et al. have also reported effective results associated with volar plate fixation without the use of bone grafting [51, 52]. Tarallo et al. concluded that this should be the preferred technique when correcting extra-articular distal radius fracture malunions, especially in elderly patients with poor bone quality [51].

\section{Infection}

Patients suspected of an infection after a fracture of the distal radius normally present with symptoms of redness, erythema, drainage, pain, swelling, tenderness, or a combination of these factors. Infection can occur as a result of k-wire fixation, external fixation or ORIF. The rate of infection after k-wire fixation has been found to be higher compared to the rate of infection after external fixation of a distal radius fracture, $33 \%$ and $21 \%$ respectively [53, 54]. Recent literature has also reported a large difference in the rate of infection between external fixation and ORIF. Esposito et al. reported a pin tract infection rate of $9.8 \%$ in patients treated with external fixation, compared to a rate of $2.8 \%$ in the ORIF group [55]. Furthermore, a meta-analysis of 1520 surgically treated distal radius fractures reported an infection rate of $11 \%$ for fractures treated with external fixation, and only a $0.8 \%$ infection rate in patients treated with internal fixation [56]. Before choosing a distal radius fracture treatment method, it is important to take these infection rates into consideration.

In addition to careful consideration of the possible treatment options, certain preventive measures can be taken to ensure patients do not develop an infection following treatment of a distal radius fracture. If possible, all pins and k-wires should be buried under the skin. A study performed by Hargreaves et al. demonstrated a significantly higher pin tract infection rate in k-wires that were left percutaneously compared to those buried under the skin [53]. The rate of infection was found to be $34 \%$ for pins left out of the skin, compared to only $7 \%$ 
when the k-wires were buried beneath the skin [53]. A majority of the infection complications seen in our facility after treatment of a distal radius fracture resulted from percutaneous pinning of the fracture (Figure 3). One specific case of pin infection observed after percutaneous pinning resulted in further complication requiring an additional surgery. The patient's pins were removed prematurely due to the infection, and as a result the patient experienced dorsal angulation of the distal radius fragment and a loss of both the radial and ulnar tilt. A corrective osteotomy was performed along with the placement of a plate to promote accurate bone re-formation. If the infection requires the removal of the pin, patients should be monitored closely for symptoms of further complications.

Preventive measures have also been studied with the use of external fixation devices. Egol et al aimed to determine the prevalence of pin-track infection associated with external fixation for the treatment of distal radius fractures [57]. They tested the ability of a chlorhexidineimpregnated disc to provide a significant reduction in pin-track infection compared to traditional methods of pin-site care. After noting a pin-track infection rate of $19 \%$ without a significant difference between pin-site care protocols, researchers concluded that the use of hydrogen peroxide care or chlorhexidine-impregnated dressings does not reduce the rate of infection [57]. Additional wound care beyond the use of dry, sterile dressings for pin-track care is not recommended. They also noted an increased risk of pin-track complications associated with an increase in age [57]. This factor should be considered carefully when deciding on a treatment option after a fracture of the distal radius.

Open distal radius fractures can also predispose patients to infection; however, the rate of occurrence is not as common compared to other factors. Gluek et al aimed to identify factors associated with infection after open distal radius fractures and found that contamination, rather than absolute wound size, was significantly associated with infection development [58]. Researchers recommended that physicians include contamination as a factor for prognosis and that open distal radius fractures be treated with multiple debridements as part of the initial treatment plan [58]. Multiple debridements can prevent the development of infection by clearing all possible sources of wound contamination.

Researchers have tested the use of prophylactic antibiotics prior to distal radius fracture treatment; however, the value of antibiotics for elective clean cases is uncertain.

Subramanian et al. conducted a recent study on the use prophylactic antibiotics prior to kwire fixation for a distal radius fracture, and concluded that they do not advocate the use of prophylactic antibiotics as it does not affect the rate of infection [59]. Further analysis should be done to investigate the benefits of prophylactic antibiotics in order to clarify the use of this prevention measure.

Infection is a common complication following a distal radius fracture. Treatment methods may vary depending on the severity of the infection. For minor infections consisting of a slight discharge and redness around the pin site, pin removal is not normally required [60, 61]. The pins and wound should be cleaned and monitored for the addition of symptoms of tenderness in the soft tissue. Patients with an infection involving the soft tissue should be given antibiotics and pins should be removed if the infection fails to improve [60, 61]. Patients must be monitored closely with frequent follow-ups and radiographic images should 
be taken to rule out a possible osteomyelitis involving the bone. In the case of osteomyelitis, surgical intervention is required [41].

\section{Tendon Complications}

Surgical fixation after a distal radius fracture can result in both flexor and extensor tendon complications. Complications may be minor such as simple irritation, adhesion formation or tenosynovitis, or more severe including laceration or rupture [5]. Tendon ruptures normally occur at a mean of 7 weeks, but can also occur within the first few weeks of a distal radius fracture [62]. Rupture complication rates can vary, and often depend on the surgical fixation technique. Margaliot et al. compared the risk of tendon complications between internal fixation and external fixation devices, finding a 6 fold higher risk of tendon rupture associated with internal fixation after a distal radius fracture [56]. Volar plate fixation was developed as an alternative to external fixation and dorsal plating. Studies have demonstrated a lower rate of tendon complications associated with volar plating, but rates have still been reported as high as $16 \%$ [63].

\section{Extensor Tendon Complications}

Extensor tendon complications can result from both volar and dorsal fixation of a distal radius fracture. The incidence of extensor tendon injuries after volar plate fixation specifically has been reported to be between $3 \%$ and 5\% [63, 64]. Complications with volar plating are likely to occur because of drill-bit penetration or dorsal screw prominence and most commonly affect the extensor pollicis longus (EPL) tendon, especially at the location of the Lister tubercle [65-67]. This is because the Lister tubercle is a watershed area of blood supply to the tendon $[66,67]$.

If tendon irritation is suspected, removal of the plate is recommended to prevent further damage. In cases of EPL rupture, in order to restore thumb function the extensor indicis proprius (EIP) may be transferred to the EPL or free tendon graft (FTG) reconstruction may be performed [5]. Both are acceptable methods to treat EPL ruptures, as shown in a recent study by Schaller et al comparing the two techniques. At a mean of 4.3 years after the surgery, the investigators noted very good to good Geldmacher scores in $86 \%$ of patients treated with EIP to EPL transfer ( $\mathrm{n}=28$ ), and $77 \%$ of patients treated with FTG reconstruction $(n=17)$ [68]. To avoid extensor tendon injuries after the use of volar locking plates, drilling should reach the dorsal cortex but not extend through it, and shorter, unicortical screws or small pegs should be used (Figure 4) [5, 65].

Extensor tendon irritation or rupture after a distal radius fracture treated with dorsal plate fixation is likely to occur due to screw-head or plate prominence [65]. Rozental et al. studied 19 patients treated with dorsal plate fixation, noting 7 cases of extensor tendon irritation and 1 rupture of the extensor digitorum communis (EDC) to the ring finger [69]. After immediate removal of the hardware, the investigators discovered complete resolution of the tendon irritation and that function was fully recovered in the affected digit after EDC tendon reconstruction [69]. 
The use of local tissue interposition flaps have been proposed as a preventive technique to minimize the risk of extensor tendon complications associated with dorsal plate fixation [5]. The flaps serve to protect the overlying tendons from the dorsal plate, preventing any contact. Altuasen and Szabo studied the use of local tissue interposition flaps, specifically the elevation of the dorsal distal radius periosteum with the third and fourth extensor compartments and subsequent re-approximation of the periosteum over the dorsal plate [70]. The investigators recommended the use of this technique after reporting no incidence of tenosynovitis, tendon adhesions, or tendon ruptures [70]. Use of the extensor retinaculum to cover the dorsal hardware has also been proposed as a possible prevention technique [71]. However, symptoms of continued dorsal wrist pain were reported in 12 of 20 patients studied by Chiang et al. after implementing this technique [71].

\section{Flexor Tendon Complications}

Flexor tendon injuries are thought to be caused primarily by hardware irritation resulting from distally placed volar locking plates [63]. The flexor pollicis longus (FPL) is the most commonly injured tendon, however the flexor digitorum superficialis, flexor digitorum profundus, and flexor carpi radialis are also at risk [72]. To avoid flexor tendon complications, volar plates should be placed in alignment with the transverse ridge along the distal volar surface of the radius between the pronator quadratus and volar radiocarpal ligaments called the watershed line $[63,65,73]$. Arora and colleagues recommend the use of a plate system with 2 distal rows of screw holes permitting screw fixation at various angles to help place the plate proximal to this line [63].

Plates placed distal to the watershed line may result in direct plate or screw head contact with the deep flexor tendons causing damaging complications [47, 73]. Soong et al recently studied the variation in volar plate placement around the watershed line and its association with flexor tendon ruptures. Through this study they developed a system for categorizing volar plate placement. For example, plates that did not extend volar to the watershed line were labeled as Grade 0 [74]. Plates volar to the watershed line, but proximal to the volar rim were labeled as Grade 1, and plates directly on or distal to the volar rim were labeled as Grade 2. Grade 2 plate prominence after use of the Acu-Loc volar plate was seen in $63 \%$ of patients and was associated with 2 of the 3 reported cases of flexor tendon ruptures [74]. Researchers concluded that direct visualization may be beneficial in order to avoid improper plate position, loss of reduction, and screw head prominence; factors directly responsible for tendon complications [74].

A study performed by Arora and colleagues on the complications associated with volar plate fixation reported 11 patients with plates placed distal to the watershed line [63]. All of the patients presented with tendon injuries including 9 cases of flexor tendon synovitis and 2 FPL ruptures. In order to prevent possible tendon complications, volar plates should be removed early if they are found to be placed distal to this line, or if the distal edge of the plate becomes prominent because of collapse of the fracture site [75]. Tada et al performed early plate removal in 9 out of 12 patients complaining of FPL tendon irritation, and concluded that volar plate removal may prevent FPL rupture [76]. 
Early removal after volar plate fixation has also been recommended for patients using steroids [75]. Bell et al experienced four patients with FPL ruptures after volar plating of a distal radius fracture and noted that all four of the patients were taking steroids [75]. In the case of FPL rupture, reconstruction can be performed using direct repair, tendon transfer, or interposition grafting [5]. Reconstruction is often performed using a palmaris longus tendon graft [63]. In the absence of the palmaris longus, however, the plantaris or long toe extensor tendons may be used [77].

To prevent the risk of flexor tendon injuries, Orbay recommends covering the volar plate with the pronator quadratus muscle [44]. Restoration of the pronator quadratus to its original position can prevent irritation of the flexor tendons by disallowing the plate from coming in contact with the tendons. However, a recent study performed by Brown and Lifchez contradicts Orbay's recommendation. The authors reported a partial-thickness laceration of the FPL 2.5 years after volar plating of a DRF, despite the restoration of the pronator quadratus to its native position [78]. They concluded that even after coverage of the volar plate with the pronator quadratus tendon, irritation can still occur and that this technique does not protect the overlying flexor tendons [78]. Further investigation should be performed to clarify the use of this prevention method.

\section{Conclusion}

Prevention of the possible complications that can occur following treatment of a distal radius fracture should be the surgeon's foremost concern. Patients should be well informed about the symptoms associated with these complications and monitored closely with follow-up appointments. Complications that do arise should be treated as early as possible to prevent further long-term consequences. This paper discussed the prevention and management of common complications however, the surgeon should be aware of all possible complications including those that were not referenced in this paper.

\section{Acknowledgments}

Research reported in this publication was supported by the National Institute of Arthritis and Musculoskeletal and Skin Diseases of the National Institutes of Health under Award Number 2 K24-AR053120-06. The content is solely the responsibility of the authors and does not necessarily represent the official views of the National Institutes of Health.

\section{References}

1. Ilyas AM, Jupiter JB. Distal radius fractures--classification of treatment and indications for surgery. Orthop Clin North Am. 2007; 38(2):167-73. v. [PubMed: 17560399]

2. Davis DI, Baratz M. Soft Tissue Complications of Distal Radius Fractures. Hand Clinics. 2010; 26(2):229-+. [PubMed: 20494749]

3. Schneppendahl J, Windolf J, Kaufmann RA. Distal radius fractures: current concepts. J Hand Surg Am. 2012; 37(8):1718-25. [PubMed: 22763062]

4. McKay SD, et al. Assessment of complications of distal radius fractures and development of a complication checklist. J Hand Surg Am. 2001; 26(5):916-22. [PubMed: 11561246]

5. Rhee PC, Dennison DG, Kakar S. Avoiding and treating perioperative complications of distal radius fractures. Hand Clin. 2012; 28(2):185-98. [PubMed: 22554662] 
6. Chung KC, Kotsis SV, Kim HM. Predictors of functional outcomes after surgical treatment of distal radius fractures. J Hand Surg Am. 2007; 32(1):76-83. [PubMed: 17218179]

7. Li Z, et al. Complex regional pain syndrome after hand surgery. Hand Clin. 2010; 26(2):281-9. [PubMed: 20494753]

8. Jellad A, Salah S, Ben Salah Frih Z. Complex regional pain syndrome type I: incidence and risk factors in patients with fracture of the distal radius. Arch Phys Med Rehabil. 2014; 95(3):487-92. [PubMed: 24080349]

9. Atkins RM, Duckworth T, Kanis JA. Features of algodystrophy after Colles' fracture. J Bone Joint Surg Br. 1990; 72(1):105-10. [PubMed: 2298766]

10. Dijkstra PU, et al. Incidence of complex regional pain syndrome type I after fractures of the distal radius. Eur J Pain. 2003; 7(5):457-62. [PubMed: 12935798]

11. Roh YH, et al. Factors associated with complex regional pain syndrome type I in patients with surgically treated distal radius fracture. Arch Orthop Trauma Surg. 2014; 134(12):1775-81. [PubMed: 25311113]

12. Combalia A. Over-distraction of the radiocarpal and midcarpal joints with external fixation of comminuted distal radial fractures. J Hand Surg Br. 1996; 21(2):289. [PubMed: 8732426]

13. Field J, Protheroe DL, Atkins RM. Algodystrophy after Colles fractures is associated with secondary tightness of casts. J Bone Joint Surg Br. 1994; 76(6):901-5. [PubMed: 7983115]

14. Zyluk A, Puchalski P. Complex regional pain syndrome of the upper limb: a review. Neurol Neurochir Pol. 2014; 48(3):200-5. [PubMed: 24981185]

15. Zyluk A. Complex regional pain syndrome type I. Risk factors, prevention and risk of recurrence. J Hand Surg Br. 2004; 29(4):334-7. [PubMed: 15234496]

16. An HS, Hawthorne KB, Jackson WT. Reflex sympathetic dystrophy and cigarette smoking. J Hand Surg Am. 1988; 13(3):458-60. [PubMed: 3379291]

17. Galer BS, Bruehl S, Harden RN. IASP diagnostic criteria for complex regional pain syndrome: a preliminary empirical validation study. International Association for the Study of Pain. Clin J Pain. 1998; 14(1):48-54. [PubMed: 9535313]

18. Patterson RW, et al. Complex regional pain syndrome of the upper extremity. J Hand Surg Am. 2011; 36(9):1553-62. [PubMed: 21872098]

19. Lee GW, Weeks PM. The role of bone scintigraphy in diagnosing reflex sympathetic dystrophy. $\mathrm{J}$ Hand Surg Am. 1995; 20(3):458-63. [PubMed: 7642927]

20. Zollinger PE, et al. Can vitamin $\mathrm{C}$ prevent complex regional pain syndrome in patients with wrist fractures? A randomized, controlled, multicenter dose-response study. J Bone Joint Surg Am. 2007; 89(7):1424-31. [PubMed: 17606778]

21. Zollinger PE, et al. Effect of vitamin $\mathrm{C}$ on frequency of reflex sympathetic dystrophy in wrist fractures: a randomised trial. Lancet. 1999; 354(9195):2025-8. [PubMed: 10636366]

22. Shah AS, Verma MK, Jebson PJ. Use of oral vitamin C after fractures of the distal radius. J Hand Surg Am. 2009; 34(9):1736-8. [PubMed: 19896015]

23. Lichtman DM, et al. Treatment of distal radius fractures. J Am Acad Orthop Surg. 2010; 18(3): 180-9. [PubMed: 20190108]

24. Ekrol I, et al. The influence of vitamin $\mathrm{C}$ on the outcome of distal radial fractures: a double-blind, randomized controlled trial. J Bone Joint Surg Am. 2014; 96(17):1451-9. [PubMed: 25187584]

25. Goris RJ. Treatment of reflex sympathetic dystrophy with hydroxyl radical scavengers. Unfallchirurg. 1985; 88(7):330-2. [PubMed: 3931223]

26. Livingstone JA, Atkins RM. Intravenous regional guanethidine blockade in the treatment of posttraumatic complex regional pain syndrome type 1 (algodystrophy) of the hand. J Bone Joint Surg Br. 2002; 84(3):380-6. [PubMed: 12002497]

27. Paraskevas KI, et al. Treatment of complex regional pain syndrome type I of the hand with a series of intravenous regional sympathetic blocks with guanethidine and lidocaine. Clin Rheumatol. 2006; 25(5):687-93. [PubMed: 16333562]

28. Bushnell BD, Bynum DK. Malunion of the distal radius. J Am Acad Orthop Surg. 2007; 15(1):2740. [PubMed: 17213380] 
29. Prommersberger KJ, et al. Malunion of the distal radius. Arch Orthop Trauma Surg. 2012; 132(5): 693-702. [PubMed: 22294090]

30. Haase SC, Chung KC. Management of malunions of the distal radius. Hand Clin. 2012; 28(2):20716. [PubMed: 22554664]

31. Sharma H, et al. Outcomes and complications of fractures of distal radius (AO type B and C): volar plating versus nonoperative treatment. J Orthop Sci. 2014; 19(4):537-44. [PubMed: 24668311]

32. Jenkins NH, Mintowt-Czyz WJ. Mal-union and dysfunction in Colles' fracture. J Hand Surg Br. 1988; 13(3):291-3. [PubMed: 3171296]

33. Prommersberger KJ, et al. Rotational deformity in malunited fractures of the distal radius. J Hand Surg Am. 2004; 29(1):110-5. [PubMed: 14751113]

34. Fernandez DL. Malunion of the distal radius: current approach to management. Instr Course Lect. 1993; 42:99-113. [PubMed: 8463707]

35. Jupiter JB, Ruder J, Roth DA. Computer-generated bone models in the planning of osteotomy of multidirectional distal radius malunions. J Hand Surg Am. 1992; 17(3):406-15. [PubMed: 1613212]

36. Athwal GS, et al. Computer-assisted distal radius osteotomy. J Hand Surg Am. 2003; 28(6):951-8. [PubMed: 14642510]

37. Miyake J, et al. Distal radius osteotomy with volar locking plates based on computer simulation. Clin Orthop Relat Res. 2011; 469(6):1766-73. [PubMed: 21203873]

38. Sato K, et al. Corrective osteotomy for volarly malunited distal radius fracture. J Hand Surg Am. 2009; 34(1):27-33. 33 e1. [PubMed: 19121727]

39. Jupiter JB, Ring D. A comparison of early and late reconstruction of malunited fractures of the distal end of the radius. J Bone Joint Surg Am. 1996; 78(5):739-48. [PubMed: 8642031]

40. Wada $\mathrm{T}$, et al. Simultaneous radial closing wedge and ulnar shortening osteotomy for distal radius malunion. Tech Hand Up Extrem Surg. 2005; 9(4):188-94. [PubMed: 16340579]

41. Turner RG, Faber KJ, Athwal GS. Complications of distal radius fractures. Orthop Clin North Am. 2007; 38(2):217-28. vi. [PubMed: 17560404]

42. Koval KJ, et al. Fractures of the distal part of the radius. The evolution of practice over time. Where's the evidence? J Bone Joint Surg Am. 2008; 90(9):1855-61. [PubMed: 18762644]

43. Chung KC, Shauver MJ, Birkmeyer JD. Trends in the United States in the treatment of distal radial fractures in the elderly. J Bone Joint Surg Am. 2009; 91(8):1868-73. [PubMed: 19651943]

44. Orbay JL. The treatment of unstable distal radius fractures with volar fixation. Hand Surg. 2000; 5(2):103-12. [PubMed: 11301503]

45. Fernandez DL. Should anatomic reduction be pursued in distal radial fractures? J Hand Surg Br. 2000; 25(6):523-7. [PubMed: 11106512]

46. Baratz ME, et al. Displaced intra-articular fractures of the distal radius: the effect of fracture displacement on contact stresses in a cadaver model. J Hand Surg Am. 1996; 21(2):183-8. [PubMed: 8683044]

47. Orbay J. Volar plate fixation of distal radius fractures. Hand Clin. 2005; 21(3):347-54. [PubMed: 16039446]

48. Tiren D, Vos DI. Correction osteotomy of distal radius malunion stabilised with dorsal locking plates without grafting. Strategies Trauma Limb Reconstr. 2014; 9(1):53-8. [PubMed: 24610600]

49. Dimitriou R, et al. Complications following autologous bone graft harvesting from the iliac crest and using the RIA: a systematic review. Injury. 2011; 42 Suppl 2:S3-15. [PubMed: 21704997]

50. Simic PM, et al. Treatment of distal radius fractures with a low-profile dorsal plating system: an outcomes assessment. J Hand Surg Am. 2006; 31(3):382-6. [PubMed: 16516731]

51. Tarallo L, et al. Volar plate fixation for the treatment of distal radius fractures: analysis of adverse events. J Orthop Trauma. 2013; 27(12):740-5. [PubMed: 23515129]

52. Mahmoud M, El Shafie S, Kamal M. Correction of dorsally-malunited extra-articular distal radial fractures using volar locked plates without bone grafting. J Bone Joint Surg Br. 2012; 94(8):10906. [PubMed: 22844051] 
53. Hargreaves DG, Drew SJ, Eckersley R. Kirschner wire pin tract infection rates: a randomized controlled trial between percutaneous and buried wires. J Hand Surg Br. 2004; 29(4):374-6. [PubMed: 15234503]

54. Ahlborg HG, Josefsson PO. Pin-tract complications in external fixation of fractures of the distal radius. Acta Orthop Scand. 1999; 70(2):116-8. [PubMed: 10366908]

55. Esposito J, et al. External fixation versus open reduction with plate fixation for distal radius fractures: a meta-analysis of randomised controlled trials. Injury. 2013; 44(4):409-16. [PubMed: 23298757]

56. Margaliot Z, et al. A meta-analysis of outcomes of external fixation versus plate osteosynthesis for unstable distal radius fractures. J Hand Surg Am. 2005; 30(6):1185-99. [PubMed: 16344176]

57. Egol KA, et al. Treatment of external fixation pins about the wrist: a prospective, randomized trial. J Bone Joint Surg Am. 2006; 88(2):349-54. [PubMed: 16452747]

58. Glueck DA, Charoglu CP, Lawton JN. Factors associated with infection following open distal radius fractures. Hand (N Y). 2009; 4(3):330-4. [PubMed: 19194762]

59. Subramanian $P$, et al. Complications of Kirschner-wire fixation in distal radius fractures. Tech Hand Up Extrem Surg. 2012; 16(3):120-3. [PubMed: 22913989]

60. Hargreaves DG, et al. The role of biofilm formation in percutaneous Kirschner-wire fixation of radial fractures. J Hand Surg Br. 2002; 27(4):365-8. [PubMed: 12162980]

61. Santy J. A review of pin site wound infection assessment criteria. International Journal of Orthopaedic and Trauma Nursing. 2010; 14(3):125-131.

62. Bonatz E, Kramer TD, Masear VR. Rupture of the extensor pollicis longus tendon. Am J Orthop (Belle Mead NJ). 1996; 25(2):118-22. [PubMed: 8640381]

63. Arora R, et al. Complications following internal fixation of unstable distal radius fracture with a palmar locking-plate. J Orthop Trauma. 2007; 21(5):316-22. [PubMed: 17485996]

64. Soong M, et al. Fracture of the distal radius: risk factors for complications after locked volar plate fixation. J Hand Surg Am. 2011; 36(1):3-9. [PubMed: 21193123]

65. Meyer C, et al. Complications of distal radial and scaphoid fracture treatment. J Bone Joint Surg Am. 2013; 95(16):1517-26. [PubMed: 23965705]

66. Kozin SH, Wood MB. Early soft-tissue complications after fractures of the distal part of the radius. J Bone Joint Surg Am. 1993; 75(1):144-53. [PubMed: 8419384]

67. Owers KL, et al. Ultrasound changes in the extensor pollicis longus tendon following fractures of the distal radius--a preliminary report. J Hand Surg Eur Vol. 2007; 32(4):467-71. [PubMed: $17566618]$

68. Schaller P, Baer W, Carl HD. Extensor indicis-transfer compared with palmaris longus transplantation in reconstruction of extensor pollicis longus tendon: a retrospective study. Scand J Plast Reconstr Surg Hand Surg. 2007; 41(1):33-5. [PubMed: 17484183]

69. Rozental TD, Beredjiklian PK, Bozentka DJ. Functional outcome and complications following two types of dorsal plating for unstable fractures of the distal part of the radius. J Bone Joint Surg Am. 2003; 85-A(10):1956-60. [PubMed: 14563804]

70. Althausen PL, Szabo RM. Coverage of distal radius internal fixation and wrist fusion devices with AlloDerm. Tech Hand Up Extrem Surg. 2004; 8(4):266-8. [PubMed: 16518102]

71. Chiang PP, Roach S, Baratz ME. Failure of a retinacular flap to prevent dorsal wrist pain after titanium Pi plate fixation of distal radius fractures. J Hand Surg Am. 2002; 27(4):724-8. [PubMed: 12132102]

72. Trevor W, Oren M, Jennifer M, Wolf MD. Soft-Tissue Complications Associated With Distal Radius Fractures. Operative Techniques in Orthopaedics. 2009:100-106.

73. Orbay JL, Touhami A. Current concepts in volar fixed-angle fixation of unstable distal radius fractures. Clin Orthop Relat Res. 2006; 445:58-67. [PubMed: 16505728]

74. Soong M, et al. Volar locking plate implant prominence and flexor tendon rupture. J Bone Joint Surg Am. 2011; 93(4):328-35. [PubMed: 21239658]

75. Bell JS, Wollstein R, Citron ND. Rupture of flexor pollicis longus tendon: a complication of volar plating of the distal radius. J Bone Joint Surg Br. 1998; 80(2):225-6. [PubMed: 9546448] 
76. Tada K, et al. Prevention of flexor pollicis longus tendon rupture after volar plate fixation of distal radius fractures. Hand Surg. 2011; 16(3):271-5. [PubMed: 22072459]

77. Unglaub F, et al. Two-staged reconstruction of the flexor pollicis longus tendon. J Hand Surg Br. 2006; 31(4):432-5. [PubMed: 16618523]

78. Brown EN, Lifchez SD. Flexor pollicis longus tendon rupture after volar plating of a distal radius fracture: pronator quadratus plate coverage may not adequately protect tendons. Eplasty. 2011; 11:e43. [PubMed: 22096615] 


\section{Key Points}

1. Prevention of the possible complications associated with a fracture of the distal radius should be the treating surgeon's primary concern

2. Complication type may vary depending on the method of treatment

3. Complication rates can depend on patient factors including patient lifestyle, age, social support and medical comorbidities

4. Early diagnosis and treatment is important to avoid possible long-term consequences 


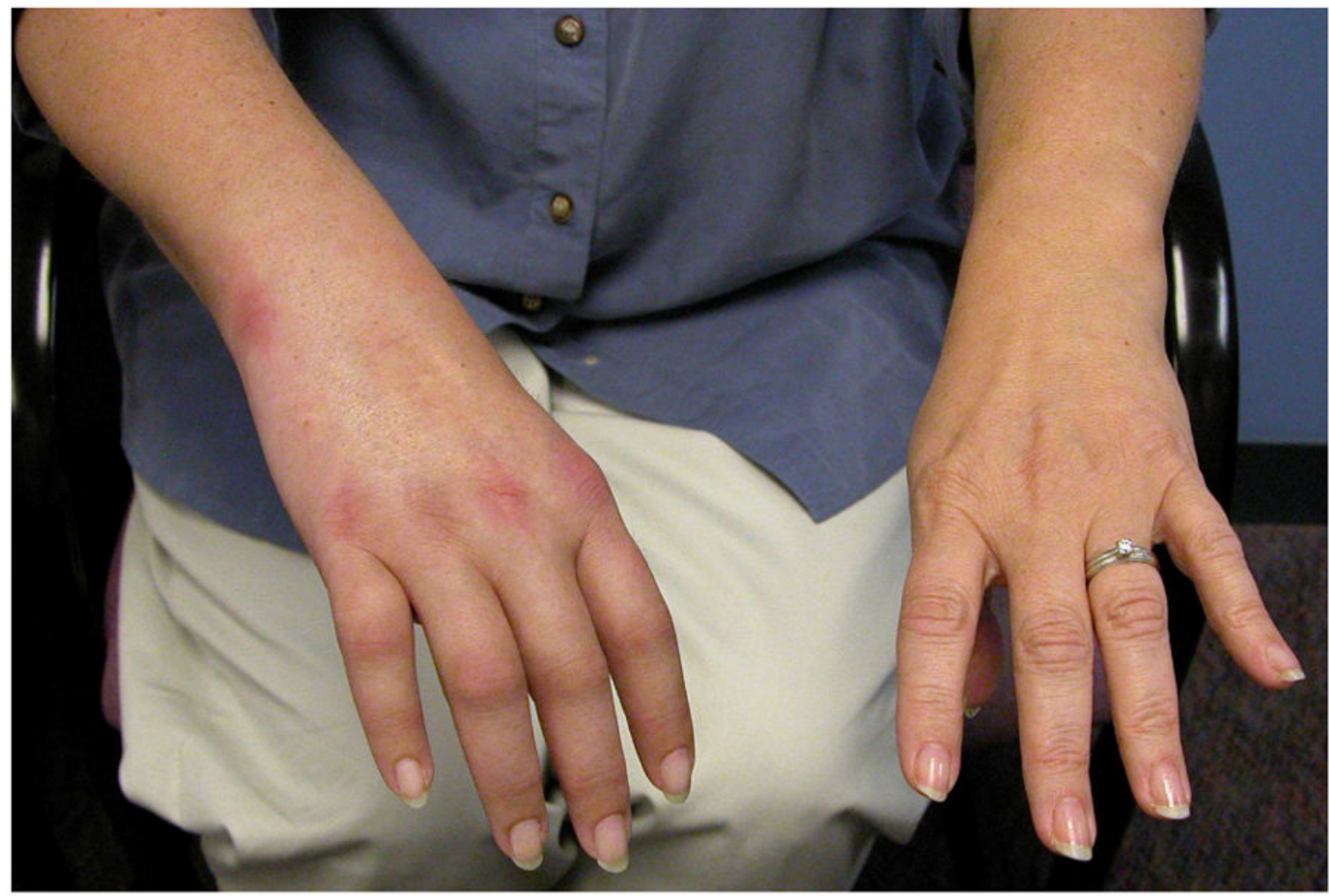

Figure 1.

Patient diagnosed with complex regional pain syndrome presenting with a shiny appearance of the right hand. 


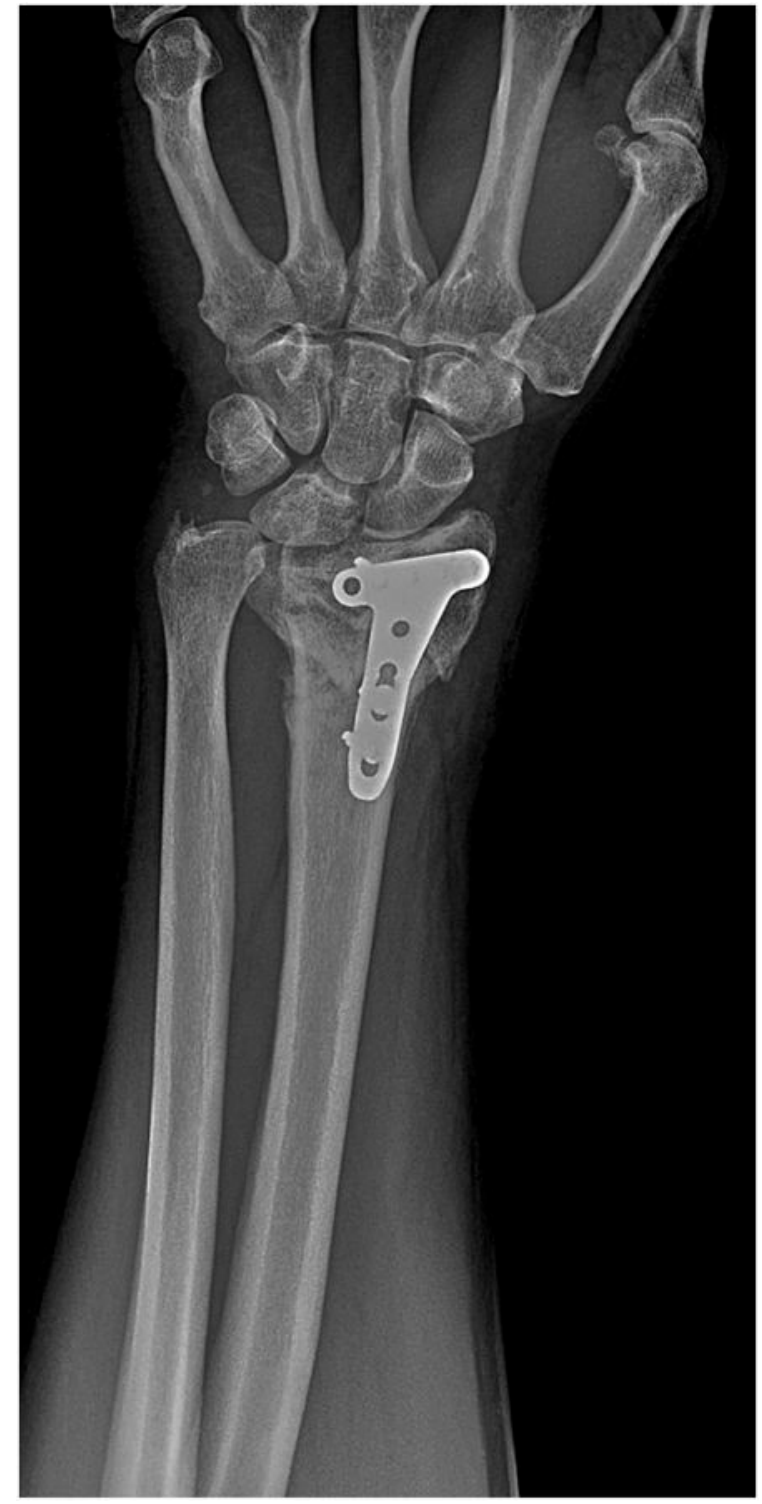

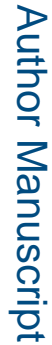

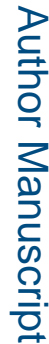

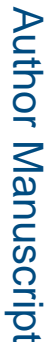




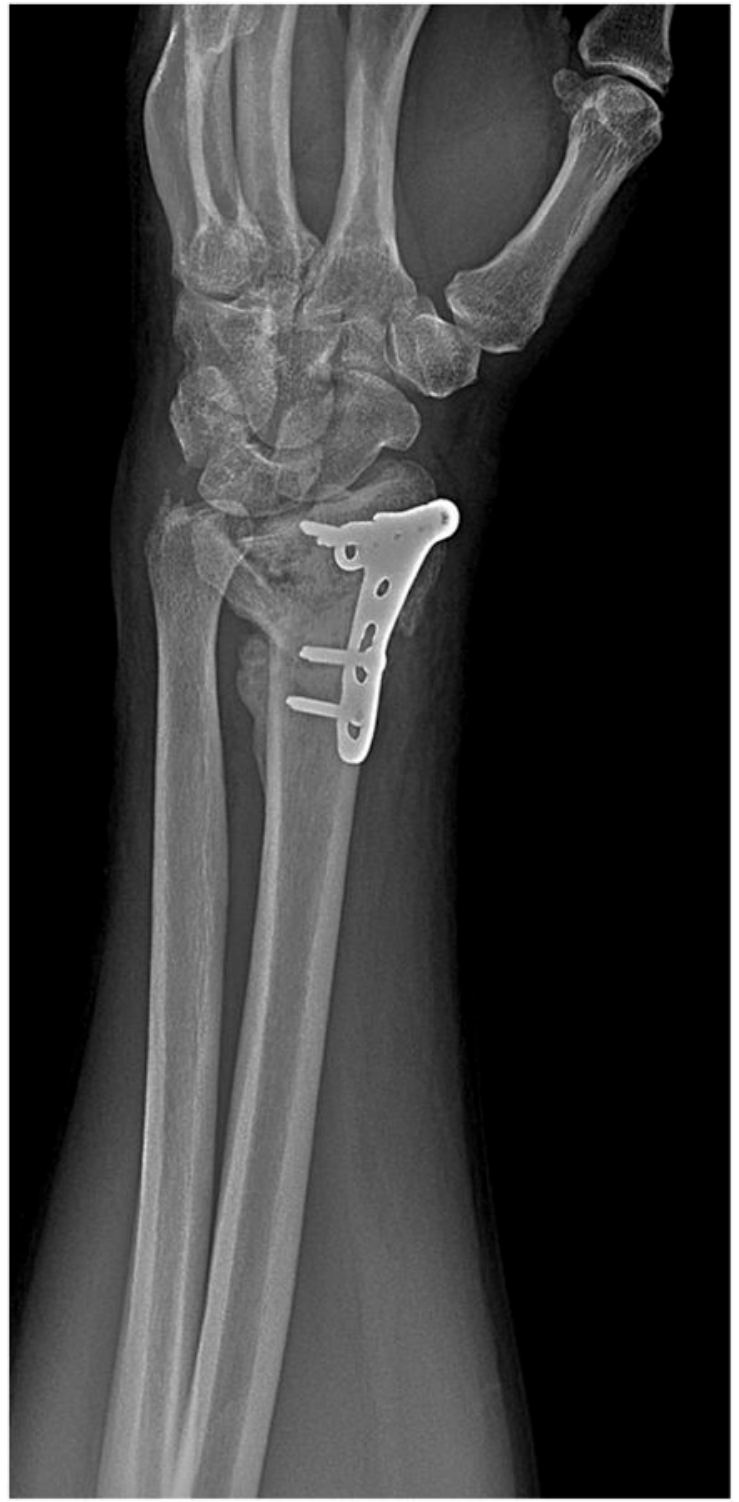

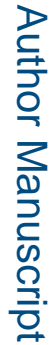

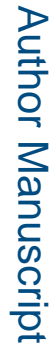

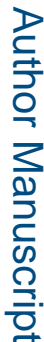




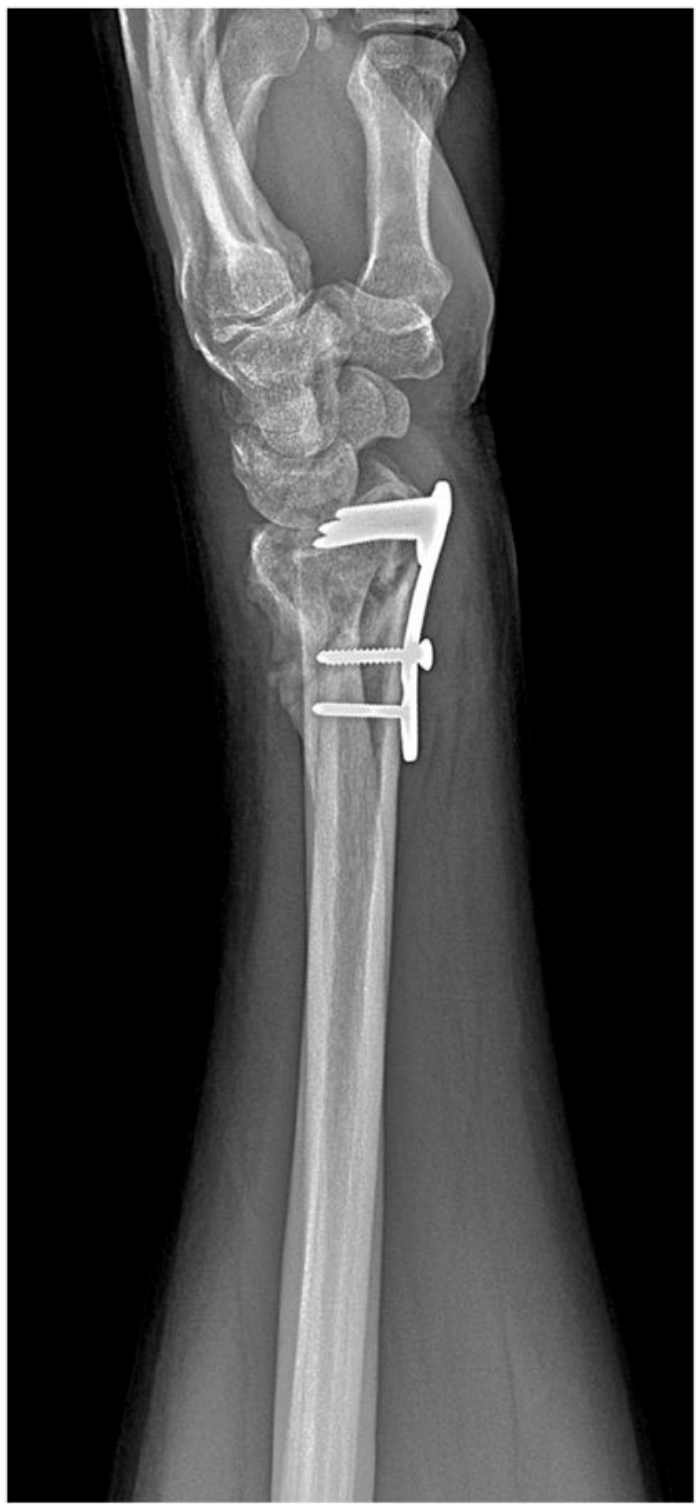

Figure 2.

a) Anteroposterior, b) Lateral and c) Oblique pre-operative $\mathrm{x}$-rays of a patient who sustained a left intra-articular distal radius fracture. The fracture was fixated at an outside hospital; however, the articular surface collapsed causing DRUJ incongruity. 


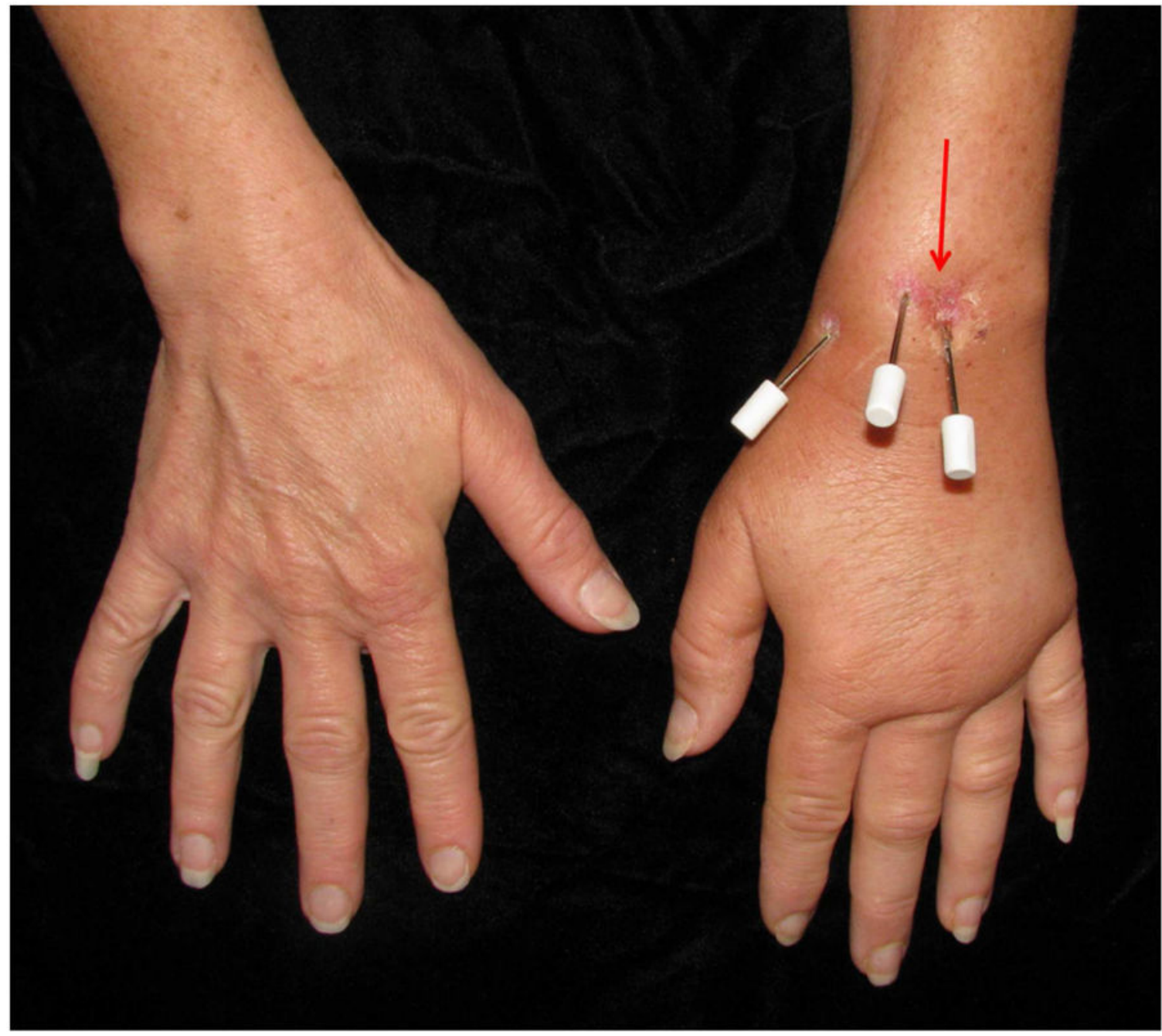

Figure 3.

Patient presenting with purulent drainage from one of the dorsal pins, which resulted in pin removal. 


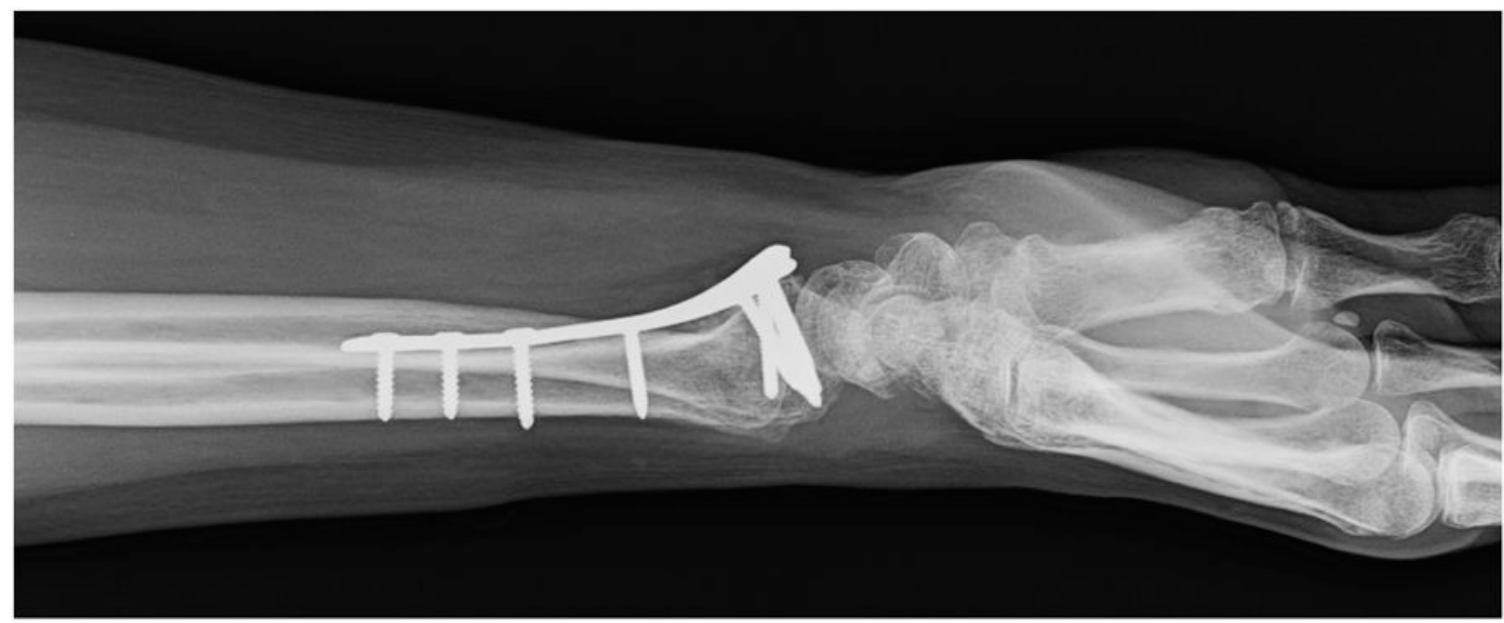

Figure 4.

Note the placement of the dorsal screws which do not penetrate the dorsal cortex, but rest $2 \mathrm{~mm}$ under the dorsal cortex to prevent tendon injury. Also note the placement of the volar plate distally over the watershed line, which can cause irritation and possible rupture of the FPL. 